\title{
Cirurgia cardíaca fetal: características hemodinâmicas da placenta durante circulação extracorpórea
}

\author{
Renato S. ASSAD*, Fan-Yen LEE*, Kim BERGER*, Frank I. HANLEY*
}

RBCCV $44205-130$

\begin{abstract}
ASSAD, R. S.; LEE, F. Y.; BERGER, K.; HANLEY, F. I. - Cirurgia cardiaca fetal: características hemodinâmicas da placenta durante circulaçăo extracorpórea. Rev. Bras. Cir. Cardiovasc., 6(1): 38-44, 1991.

RESUMO: A diminuição da perfusão placentária com conseqüente distúrbio de trocas gasosas ocorre com freqüência após a circulação extracorpórea (CEC) fetal experimental. Com o objetivo de caracterizar a hemodinâmica placentária durante a CEC, foram colocadas em CEC sete placentas de ovelhas, isoladas in situ, através da canulação dos vasos umbilicais. O fluxo da CEC foi alterado de 15 a $300 \mathrm{ml} / \mathrm{min} / \mathrm{kg}$ de peso fetal, em normotermia e hipotermia. A resistência vascular placentária (RVP) permaneceu constante durante uma pressão de perfusão e fluxo da CEC acima de $40 \mathrm{mmHg}$ e $150 \mathrm{ml} / \mathrm{min} / \mathrm{kg}$, respectivamente. Abaixo destes valores, a relação da RVP com estes dois parâmetros foi inversamente proporcional. Um maior aumento da RVP foi observado durante a hipotermia. A implicação clínica destes achados reside no fato de que a diminuição do fluxo e da pressão de perfusāo placentária durante a CEC pode conduzir a um ciclo vicioso, resultando em prejuizo adicional da perfusão placentária das trocas gasosas, sendo que a hipotermia agrava ainda mais esta disfunção placentária.
\end{abstract}

DESCRITORES: cirurgia cardiaca fetal; placenta, hemodinâmica durante circulação extracorpórea.

\section{INTRODUÇÃO}

A correção cirúrgica intra-uterina de certas cardiopatias congênitas pode proporcionar significante vantagem terapêutica em relação aos métodos terapêuticos convencionais pós-natais. Tal abordagem exige o domínio não apenas da resposta fetal ao estresse cirúrgico, como também da fisiopatologia da circulação extracorpórea (CEC) fetal. Estudos experimentais sobre a resposta fetal ao estresse cirúrgico e à CEC apontam a placenta como a maior causa de morbidade ${ }^{5}$. A resistência vascular placentária (RVP) eleva-se durante e após a CEC, resultando em diminuição da perfusão placentária, distúrbios de trocas gasosas e óbito fetal ${ }^{4}$.

Este estudo foi realizado com o objetivo de analisar detalhadamente a resposta placentária à CEC, utilizan- do-se um circuito de placentas isoladas in situ, tendo em vista que a disfunção placentária após a CEC fetal parece estar relacionada com alteração da hemodinâmica placentária. Este sistema permitiu uma avaliação precisa do comportamento básico dos vasos placentários durante a CEC sob condiçōes controladas.

\section{MATERIAL E MÉTODOS}

Foram estudadas sete ovelhas grávidas com idade gestacional entre 125 a 145 dias. Os animais permaneceram em jejum de 24 a 48 horas antes da cirurgia. A anestesia foi induzida com Ketamine intramuscular (20 mg/kg) e mantida com inalação de uma mistura de $2 \%$ de halotano e $50 \%$ de óxido nitroso.

Trabalho realizado no The Children's Hospital, Departamento de Cirurgia Cardiovascular. Harvard Medical School. Boston, MA, USA. Apresentado ao 18: Congresso Nacional de Cirurgia Cardiaca. Rio de Janeiro, RJ, 5 e 6 de abril, 1991.

* Do Departamento de Cirurgia Cardiovascular do The Children's Hospital. Harvard Mecical School.

Endereço para separatas: Renato Assad. Av. Dr. Enéas de Carvalho Aguiar, 44. Divisão Cirúrgica. 05403 São Paulo, SP, Brasil. 
ASSAD, R. S.; LEE, F. Y.; BERGER, K.; HANLEY, F. I. - Cirurgia cardiaca fetal: características hemodinâmicas da placenta durante circulaçāo extracorpórea. Rev. Bras. Cir. Cardiovasc., 6(1): 38-44, 1991.

A ovelha foi colocada em decúbito dorsal horizontal. Foram realizadas laparotomia mediana intra-umbilical e histerotomia de aproximadamente $10 \mathrm{~cm}$ para se expor o feto. Algumas amostras de cotilédones foram colhidas imediatamente após a histerotomia, para se avaliar o conteúdo de água e compará-lo ao das amostras colhidas no final do experimento.

Os vasos femorais fetais foram então cateterizados, para infusāo de drogas e monitorização da pressão arterial fetal. Os vasos umbilicais foram expostos e isolados dentro da cavidade abdominal fetal através de uma laparotomia paramediana direita, para serem canulados em direção à placenta. $O$ feto foi mantido no útero, apesar do óbito fetal ocasionado pela canulação dos vasos umbilicais. A circulação umbilical foi imediatamente restabelecida através do circuito extracorpóreo, com um fluxo de $200 \mathrm{ml} / \mathrm{min} / \mathrm{kg}$ de peso fetal (Figura 1), que representa o fluxo fisiológico aproximado da artéria umbilical.

Nitroprussiato (NTP) de sódio foi utilizado em duas partes distintas do experimento. Inicialmente, esta droga foi infundida pela veia femoral fetal antes da canulação dos vasos umbilicais, com o objetivo de diminuir a pressão arterial fetal em $60 \%$ dos valores basais, inibindo, assim, o espasmo do cordão umbilical associado à manipulação de seus vasos. Posteriormente, o NTP foi infundido na linha arterial do circuito da CEC com uma dose suficiente para manter a pressão da artéria umbilical dentro dos limites fisiológicos para um fluxo de CEC de $200 \mathrm{ml} / \mathrm{min} / \mathrm{kg}$. Quando a dose ideal foi atingida, a infusão foi mantida constante, independentemente da variaçăo do fluxo placentário. $\mathrm{O}$ objetivo dessa infusão foi o de minimizar do tônus vascular placentário durante as condiçōes basais, simulando, assim, a RVP de repouso.

Após a heparinização da ovelha $(2 \mathrm{mg} / \mathrm{kg})$, foram utilizados $700 \mathrm{ml}$ de sangue venosos materno como solução de priming. Como o sangue materno apresenta o hematócrito em torno de $25 \%$, a sua utilizaçāo propor-

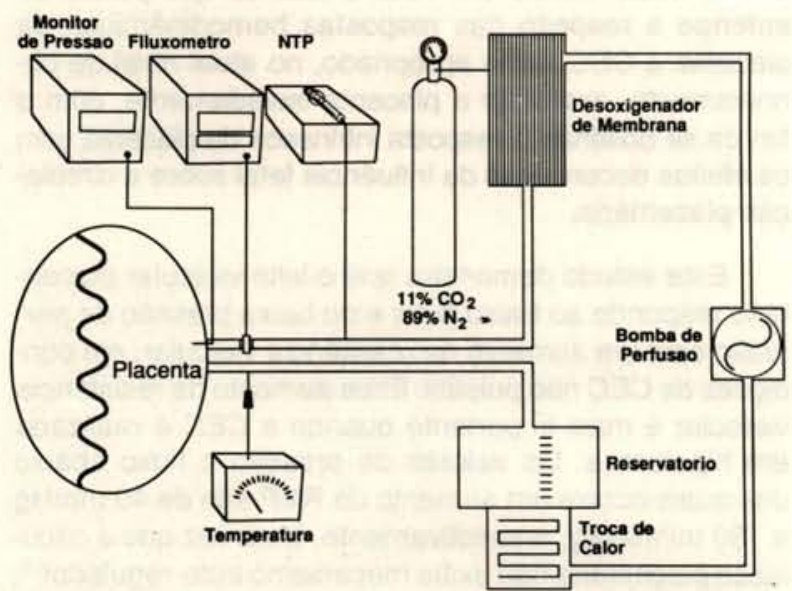

Fig. 1 - Diagrama do circuito extracorpóreo para o isolamento da placenta. (NTP $=$ Nitroprussiato). cionou uma hemodiluição moderada satisfatória para a CEC. Foi utilizado um oxigenador de membranas infantil (Cobe Laboratories, Inc., Lakewood, E.U.A.) no circuito para ajuste da temperatura, $\mathrm{pO}_{2}$ e $\mathrm{pCO}_{2}$. Um tanque composto de $5 \%$ de $\mathrm{CO}_{2}$ e $95 \%$ de $\mathrm{N}_{2}$ foi utilizado para manter a gasometria arterial dentro dos limites fisiológicos do feto.

O fluxo arterial da CEC foi medido com um transdutor eletromagnético (MVF-3100, Nihon Kohden, Tóquio, Japão) e a pressão de perfusão placentária foi medida através de um cateter ( $22 \mathrm{G}$ ) inserido no lume de uma das cânulas arteriais. O retorno venoso das veias umbilicais se fazia através da gravidade para um reservatório. Os dados de temperatura foram colhidos através de um termômetro instalado na cânula venosa.

O fluxo da CEC foi alterado de 15 a $300 \mathrm{ml} / \mathrm{min} / \mathrm{kg}$ de peso fetal. Um período mínimo de cinco minutos após o ajuste do fluxo foi estabelecido para anotação da pressão de perfusão placentária correspondente. A coleta de dados foi realizada em três condiçōes de temperatura: 1) normotermia; 2) hipotermia $\left(28^{\circ} \mathrm{C}\right)$; 3) normotermia, novamente.

A RVP foi calculada através da razão entre os dados de pressão e fluxo da artéria umbilical, de acordo com a lei de Ohm, modificada para aplicação de fluxo de líqüidos.

Os dados foram analisados estatisticamente através da regressão linear. A significância estatística foi estabelecida ao nível de $5 \%$. Os valores estão expressos em médias \pm desvio padrão das médias.

\section{RESULTADOS}

Os dados de controle da pressão arterial umbilical (pré e pós nitroprussiato, hematócrito e gasometria arterial fetal encontram-se listados na Tabela 1. Esses dados foram obtidos antes do isolamento placentário e se encontram nos limites fisiológicos de fetos ovinos $1,7,10$.

TABELA 1

DADOS DE CONTROLE DA PRESSĀO ARTERIAL FETAL, HEMATÓCRITO E GASOMETRIA ARTERIAL

\begin{tabular}{lll}
\hline Pressão arterial & Pré-NTP: & $55,6 \pm 2,9$ \\
& Pós-NTP: & $33,8 \pm 1.8$ \\
Hematócrito (\%) & & $39,0 \pm 1,2$ \\
pH & & $7,26 \pm 0,03$ \\
p02 (torr) & $21,6 \pm 0,9$ \\
pC02 (torr) & & $61,5 \pm 4,2$
\end{tabular}

Valores $=$ Média \pm Desvio Padrāo das Médias; $n=7 ;$ NTP = Nitroprussiato. 
ASSAD, R. S.; LEE, F. Y.; BERGER, K.; HANLEY, F. I. - Cirurgia cardiaca fetal: características hemodinâmicas da placenta durante circulaçāo extracorpórea. Rev. Bras. Cir. Cardiovasc., 6(1): 38-44, 1991.

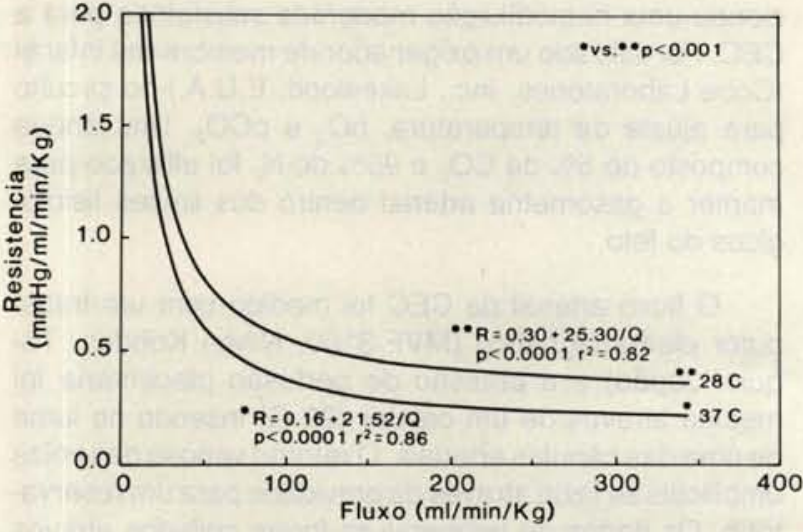

Fig. 2-Resistência vascular placentária em funçăo do fluxo da artéria umbilical, durante normotermia e hipotermia $\left(28^{\circ} \mathrm{C}\right)$.

TABELA 2

COEFIFIENTES DE CORRELAÇĀO DE RESISTÊNCIA X FLUXO DE CADA EXPERIMENTO

\begin{tabular}{cccc}
\hline \multirow{2}{*}{ Animal $n^{\circ}$} & \multicolumn{2}{c}{ Valor de $r^{2}$} & Valor de $p^{* *}$ \\
\cline { 2 - 3 } & Normotermia & Hipotermia & \\
\hline 1 & $1,00^{*}$ & $1,00^{*}$ & 0,0002 \\
2 & $0,99^{*}$ & $0,88^{*}$ & 0,0004 \\
3 & $0,99^{*}$ & $1,00^{*}$ & 0,0030 \\
4 & $0,99^{*}$ & $0,96^{*}$ & 0,0010 \\
5 & $0,99^{*}$ & $0,92^{*}$ & 0,0200 \\
6 & $0,99^{*}$ & $0,98^{*}$ & 0,9960 \\
7 & $0,95^{*}$ & $0,92^{*}$ & 0,0001 \\
\hline
\end{tabular}

Observação: * Todas as curvas foram estatisticamente significantes $(p<0,003)$. ${ }^{*}$ Normotermia versus hipotermia.

A RVP variou inversamente ao fluxo de CEC abaixo de $150 \mathrm{ml} / \mathrm{min} / \mathrm{kg}$, durante normotermia e hipotermia. Para fluxos de CEC maiores que $150 \mathrm{ml} / \mathrm{min} / \mathrm{kg}$, a RVP permanece constante durante ambas as condições de temperatura (Figura 2). Essa relação inversamente proporcional entre fluxo e resistência para valores de fluxo inferiores a $150 \mathrm{ml} / \mathrm{min} / \mathrm{kg}$ foi observada em todos os experimentos e a elevação da RVP durante a hipotermia foi estatisticamente significante em seis animais (Tabela 2).

A Figura 3 mostra a relaçāo entre pessão de perfusão placentária e RVP. De forma similar, a RVP variou inversamente com uma pressão de perfusão abaixo de $40 \mathrm{mmHg}$, permanecendo relativamente constante para valores acima de $40 \mathrm{mmHg}$. As curvas de fluxo-resistência e pressão-resistência foram desviadas para cima e para a direita durante a hipotermia, quando comparadas às curvas de normotermia, indicando uma maior RVP para um determinado fluxo. A média de aumento da RVP em hipotermia foi de $38,4 \%$ acima dos níveis de normotermia ( $p<0,001)$. A terceira curva de normotermia (não mostrada) retornou para os valores basais.

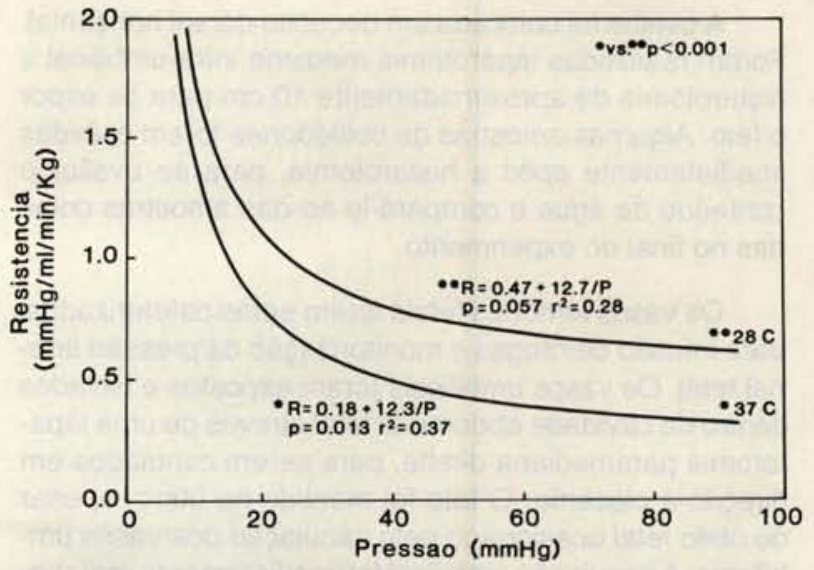

Fig. 3-Resistência vascular placentária em funçăo da pressăo da artéria umbilical, durante normotermia e hipotermia $\left(28^{\circ} \mathrm{C}\right)$.

O conteúdo de água dos cotilédones variou de 0,83 $\pm 0,01$ na condição basal para $0,86 \pm 0,01$ no final do experimento ( $p=0,03$ ). Foram observados aproximadamente $20 \%$ (variação de 0 a $43 \%$ ) de cotilédones rotos.

\section{DISCUSSÃO}

O isolamento da placenta foi desenvolvido para se estudarem detalhadamente os efeitos da CEC não pulsátil na hemodinâmica placentária, simulando, assim, a hemodinâmica presente na placenta durante a CEC fetal. O fluxo placentário foi controlado (variável dependente), enquanto que a pressão foi medida como variável independente. A resistência foi, entāo, calculada através dos dados de pressão e fluxo. Conseqüentemente, os dados deste estudo são apresentados primariamente como uma relação de fluxo-resistência. Essa relação fornece informaçōes úteis a respeito da aplicação clínica durante a CEC fetal. Tendo em vista o fato de que pouco se entende a respeito das respostas hemodinâmicas da placenta à CEC, seria apropriado, no atual nível de conhecimento, examinar a placenta isoladamente, com o fim de se observar a resposta intrínseca da placenta sem os efeitos decorrentes da influência fetal sobre a circulação placentária.

Este estudo demonstra que o leito vascular placentário responde ao baixo fluxo e/ou baixa pressão de perfusāo com um aumento na resistência vascular, em condiçōes de CEC não pulsátil. Esse aumento da resistência vascular é mais importante quando a CEC é realizada em hipotermia. Os valores de pressão e fluxo abaixo dos quais ocorre um aumento da RVP são de $40 \mathrm{mmHg}$ e $150 \mathrm{ml} / \mathrm{min} / \mathrm{kg}$, respectivamente. Uma vez que a circulação placentária não exibe mecanismo auto-regulador ${ }^{8}$, o aumento da RVP com uma pressão de perfusão e fluxo abaixo dos valores acima citados pode ser causado 
ASSAD, R. S.; LEE, F. Y.; BERGER, K.; HANLEY, F. I. - Cirurgia cardiaca fetal: características hemodinâmicas da placenta durante circulação extracorpórea. Rev. Bras. Cir. Cardiovasc., 6(1): 38-44, 1991.

pelo colapso passivo do leito vascular placentário. De acordo com os estudos agudos de BISSONETTE \& FARREL ${ }^{3}$, POWER \& LONGO ${ }^{17}$ e VETH \& VANBEMMEL ${ }^{22}$, o tecido extravascular exerce uma influência na conformação da vasculatura placentária fetal, um mecanismo denominado como "Resistência de Starling" 3, 22, ou "fenômeno de cascata placentária" ${ }^{21}$. Por outro lado, BERMAN et alii ${ }^{2}$ e THORNBURG et alii ${ }^{22}$ não apoiam a existência de qualquer relação entre a RVP e a pressão vascular uterina e vice-versa, nos estudos de fetos de ovelhas instrumentados cronicamente. A razão para diferentes achados entre todos esses estudos pode estar relacionada às condiçōes do estudo. BERMAN Jr. et alii ${ }^{2}$ e THORNBURG et alii ${ }^{21}$ utilizaram preparações cronicamente instrumentadas, sendo que o efeito da resistência de Starling não foi obsevado. O presente estudo e outros ${ }^{3,17,22}$, embora diferentes entre si, foram conduzidos de forma aguda, com um fluxo não pulsátil de CEC. Sob tais condiçōes, pode ocorrer edema importante dos cotilédones, como foi claramente demonstrado neste estudo, conforme o aumento do conteúdo de água dos cotilédones e o significante número de cotilédones rotos. Esse edema aumentaria a pressão extravascular, a qual atuaria como uma resistência de Starling. Esse achado é importante no sentido de que as condições clínicas da CEC fetal se aproximariam mais das condições do presente estudo que aquelas de estudos crônicos.

Uma vez que a pressão intersticial não foi medida nos cotilédones, nenhuma afirmativa definitiva pode ser feita a respeito do mecanismo de resistência de Starling. É possível que um mecanismo de abertura ("recruitmenf') de vasos pressão-dependente seja atuante, o que também explicaria os achados deste estudo. Esse mecanismo de abertura de vasos não explicaria, entretanto, as diferenças observadas entre os estudos agudos e crônicos.

A possibilidade de um aumento da RVP com hipofluxo da artéria umbilical ( $<150 \mathrm{ml} / \mathrm{mim} / \mathrm{kg}$ ) como resultante de baixa dose de NTP é pouco provável. O NTP foi infundido através de uma bomba separada com um fluxo constante. Portanto, durante o hipofluxo da artéria umbilical, os vasos dos cotilédones estariam recebendo maior concentração dessa droga. Caso o mecanismo de fechamento ("derecruitmenf') de vasos estivesse atuante durante o hipofluxo, parte dos vasos dos cotilédones será excluída da circulaçăo, e os vasos restantes estariam expostos a maior concentraçāo de NTP.

Os achados deste estudo podem também auxiliar no esclarecimento de outras observações. Numerosos estudos têm demonstrado que potentes vasodilatadores, como a adenosina ${ }^{11}$, hidralazina ${ }^{12}$, prostaciclina ${ }^{14,15}$ e 6-ceto-prostaglandina E1 ${ }^{19}$ causam um aumento inesperado da RVP quando administrados ao feto in vivo. A respeito da prostaciclina, que tem sido a droga mais estudada, esse fato ocorre apesar dos achados de que ela atua como um potente vasodilatador in vitro ${ }^{15}$. Para explicar esses achados, afirma-se que essas substâncias causam uma importante vasodilatação do leito vascular sistêmico periférico in vivo, com conseqüente diminuição da pressão de perfusão umbilical. Com a queda da pressão da artéria umbilical, a distensão da parede vascular placentária pode diminuir, ou pode ocorrer, ain$\mathrm{da}$, o fechamento dos vasos, causando um aumento calculado na RVP. Esse fato seria apenas observado caso a vasculatura placentária se apresentasse totalmente dilatada, de modo que pouco ou nenhum efeito direto do vasodilatador na placenta seria possível. Esses argumentos, em conjunto com os achados deste estudo, sugerem que a vasculatura placentária apresenta-se realmente próxima à vasodilatação máxima sob a condição de repouco. $O$ presente estudo documenta, pela primeira vez, o aumento do RVP decorrente da diminuição da pressão de perfusão umbilical.

A hipotermia causa um efeito negativo adicional ao fluxo sangüíneo placentário durante a CEC fetal, causando maior RVP e posterior prejuízo das trocas gasosas. Embora os dados de hipotermia tenham sido colhidos após os de normotermia, os achados durante hipotermia nāo representam degeneração da preparação, uma vez que a terceira curva de normotermia retornou para a curva normotérmica basal. O mecanismo de aumento da resistência vascular durante a hipotermia tem sido atribuído ao aumento da viscosidade sangüínea e/ou da estimulação simpática ${ }^{18}$. PARK et alii ${ }^{16}$ e SOUHRADA et alii ${ }^{20}$ demonstraram que o resfriamento do músculo liso das vias aéreas na mesma margem de temperatura do presente estudo induzia a uma resposta contrátil bifásica: 1) uma contração imediata durante 2,25 minutos; 2) posterior relaxamento lento, comumente atingindo valores menores que o tonus inicial. $\mathrm{O}$ estudo de SOUHRADA et alii ${ }^{20}$ demonstrou, também, que uma menor resposta contrátil ao resfriamenteo súbito era obtida quando o tônus inicial era diminuído. No presente estudo, o efeito direto da hipotermia na musculatura lisa vascular é improvável de ser importante estímulo para uma maior RVP, pelos seguintes motivos: 1) após atingir os $28^{\circ} \mathrm{C}$, foi estabelecido um período mínino de cinco minutos antes de se coletarem os dados; 2) o tônus vascular inicial foi minimizado pela administração de um vasodilatador direto da musculatura lisa vascular. Outros estudos de HEGNAUER et alii ${ }^{9}$ mostraram que a viscosidade aumenta significantemente em baixas temperaturas, em torno de $33 \%$ de 37 a $28^{\circ} \mathrm{C}$. No presente estudo, o aumento da RVP durante a hipotermia mostra uma alteração semelhante em relação à margem de temperatura equivalente, sugerindo que essa alteração pode ser atribuída principalmente ao aumento da viscosidade sangüinea. Além do mais, não há tecido nervoso simpático na vasculatura feto placentária ${ }^{6}$.

\section{Autocrítica do estudo}

As vantagens do isolamento placentário estāo relacionadas ao fato de que os parâmetros de pressão e 
fluxo podem ser precisamente controlados e medidos, além do que as influências procedentes do feto sobre a circulação placentária são removidos, premitindo, assim, a observação de alteraçōes intrínsecas à própria placenta. Entretanto, essas vantagens devem ser consideradas no contexto de que o isolamento de um ógão não reflete a fisiologia global do animal.

Outros aspectos da preparação merecem um breve comentário. $\mathrm{O}$ oxigenador simulou a respiração dos tecidos no circuito, removendo $\mathrm{O}_{2}$ e acrescentando $\mathrm{CO}_{2}$ ao sangue circulante, com o objetivo de fornecer uma gasometria arterial fisiológica ao leito vascular placentário. Finalmente, o uso de nitroprussiato de sódio introduz um elemento não fisiológico para esse modelo. Inicialmente, o NTP foi infundido no feto antes da manipulação do cordão umbilical, para tornar possível a canulação dos vasos. Caso contrário, ocorreria um severo vasoespasmo do cordāo que proibiria o uso desse modelo experimental. Durante a CEC da placenta, O NTP foi utilizado com a finalidade de se atingir uma condição basal de RVP em níveis relativamente normais. Em condiçōes de repouso, a vasculatura placentária apresenta-se quase totalmente dilatada ${ }^{8,13}$, com valores de resistência variando entre $0,14 \pm 0,01$ a $0,30 \pm 0,02$ $\mathrm{mmHg} / \mathrm{ml} / \mathrm{min} / \mathrm{kg}^{14,15,19}$. Neste estudo, o nitroprussiato foi administrado até que a RVP alcançasse níveis normais $(0,26 \pm 0,03 \mathrm{mmHg} / \mathrm{ml} / \mathrm{min} / \mathrm{kg})$ para um fluxo normal de artéria umbilical $(200,7 \pm 8,49 \mathrm{ml} / \mathrm{min} / \mathrm{kg})$, simulando o tônus vascular placentário normal observado nas preparaçōes crônicas sem estresse ${ }^{14,15,19}$. Uma vez que a placenta se apresenta no seu estado de vaso- dilatação máxima em condições de repouso, os valores de resistência atingidos em nossas preparaçōes utilizando NTP sugerem que os mesmos estavam próximos da vasodilatação máxima. Entretanto, esse fato não é crítico para esses experimentos. A principal finalidade do uso de NTP foi atingir condiçōes hemodinâmicas basais.

\section{CONCLUSÕES}

Uma vez que a RVP aumenta com uma pressão de perfusão e fluxo abaixo de $40 \mathrm{mmHg}$ e $150 \mathrm{ml} / \mathrm{min} / \mathrm{kg}$, respectivamente, condiçōes que levam a uma pressão e/ou fluxo de artéria umbilical abaixo desses valores críticos durante a CEC fetal poderão estar associados a uma perfusão placentária inadequada, e conseqüentemente, a distúrbios de trocas gasosas placentárias. Com isso, estabelece-se um mecanismo de "feedback negativo", o qual reduz ainda mais a perfusão placentária, isto é, quanto menor a pressão de perfusão ou o fluxo sangüíneo placentário, maior a resistência placentária.

O sucesso da cirurgia cardíaca fetal está relacionado com a observação desses parâmetros de pressão, fluxo e temperatura durante a CEC fetal, uma vez que a RVP normal é crítica para a sobrevida fetal durante e após a CEC. Esses parâmetros devem ser levados em consideração quando se planejar um esquema para a aplicação clínica da CEC fetal.

RBCCV 44205-130

ASSAD, R. S.; LEE, F. Y.; BERGER, K.; HANLEY, F. I. - Fetal cardiac surgery: hemodynamic characteristics of placenta during extracorporeal circulation. Rev. Bras. Cir. Cardiovasc., 6(1): 38-44, 1991.

ABSTRACT: Decreased placental perfusion and respiratory gas exchange have been observed following experimental fetal cardiopulmonary bypass (CPB). To better characterize placental hemodynamics during $\mathrm{CPB}, 7$ isolated in-situ lamb placentas were placed on a CPB circuit, using umbilical arterial and venous cannulation. Measures were taken to simulate normal placental hemodynamics. Perfusion flow rates were varied randomly from 15 to $300 \mathrm{ml} / \mathrm{min} / \mathrm{kg}$ fetal wt. during both normothermia and hypothermia. Placental vascular resistance (PVR) remained constant when perfusion pressure and flow were varied above 40 $\mathrm{mmHg}$ and $150 \mathrm{ml} / \mathrm{min} / \mathrm{kg}$ respectively. Below these values, PVR varied inversely. This increase in PVR was more marked when CPB was performed with hypothermia as compared to normothermia. The clinical implications is that decreased placental flow and pressure on CPB may lead to a vicious cycle resulting in further impariment of placental perfusion and respiratory gas exchange. Hypothermia promotes this impairment.

DESCRIPTORS: heart surgery, fetal; placenta, hemodynamic during extracorporeal circulation. 
ASSAD, R. S.; LEE, F. Y.; BERGER, K.; HANLEY, F. I. - Cirurgia cardiaca fetal: características hemodinâmicas da placenta durante circulação extracorpórea. Rev. Bras. Cir. Cardiovasc., 6(1): 38-44, 1991.

\section{REFERÊNCIAS BIBLIOGRÁFICAS}

1 ASSALI, N. S.; BRINKMAN, C. R.; NUWAYHID, B. - Comparison of maternal and fetal cardiovascular functions in acute and chronic experiments in the sheep. $A m$. J. Obstet. Gynecol., 120: 411-425, 1974.

2 BERMAN Jr., W.; GOODLIN, R. C.; HEYMANN, M. A.; RUDOLPH, A. M. - Relationships between pressure and flow in the umbilical and uterine circulations of the sheep. Circ. Res., 38: 262-266, 1976.

3 BISSONETTE, J. M. \& FARREL, R. C. - Pressure-flow and pressure-volume relationships in the fetal placental circulation. J. Appl. Physiol., 35: 355-360, 1973.

4 BRADLEY, S. M.; HANLEY, F. L.; JENNINGS, R. W.; DUNCAN, B. W.; JESTER, J. A.; VERRIER, E. D. - Regional blood flows during cardiopulmonary bypass in fetal lambs: the effect of nitroprusside. Circulation, 82 (Supl. 3): 413, 1990. (Resumo).

5 BRADLEY, S. M.; VERRIER, E. D.; DUNCAN, B. W.; LONGAKER, M. T.; TURLEY, K.; HARRISON, M. R.; HANLEY, F. L. - Cardiopulmonary bypass in the fetal lamb: effect of sodium nitroprusside. Circulation, 80 (Supl. 2): II-220, 1989 (Resumo).

6 CRAWFORD, J. M. - Vascular anatomy of the human placenta. Am. J. Obstet. Gynecol., 84: 1543-1567, 1962.

7 GOETZMAN, B. W.; ITSKOVITZ, J.; RUDOLPH, A. M. Fetal adaptations to spontaneous hypoxemia and responses to maternal oxygen breathing. Biol. Neonate, 46: $276-284,1984$.

8 GREISS, F. C. - Pressure-flow relationships in the gravid uterine vascular bed. Am. J. Obstet. Gynecol., 96: 41-47, 1966.

9 HEgNAUER, A. H.; SHRIBER, W. J.; HATERIUS, H. O. - Cardiovascular response of the dog to immersion hypothermia. J. Appl. Physiol., 161: 455-465, 1950.

10 IWAMOTO, H. S. \& RUDOLPH, A. M. - Effects of angiotensin II on the blood flow and its distribution fetal lambs. Circ. Res., 48: 183-189, 1981.

11 LANDAUER, M.; PHERnetTON, T. M.; RANKIN, J. H. G. - Maternal ovine placental vascular responses to adenosine. Am. J. Obstet. Gynecol., 154:1152-1155, 1986.

12 LIPSHITZ, J.; AHOKAS, R. A.; REYNOLDS, S. L. - The effect of hydralazine on placental perfusion in the spontaneously hypertensive rat. Am. J. Obstet. Gynecol., 156: 356-359, 1987.

13 PARISI, V. M. \& WALSH, S. W. - Arachidonic acid metabolities and the regulation of placental and other vascular tone during pregnancy. Sem. Perinatol., 10: 288-298, 1986.

14 PARISI, V. M. \& WALSH, S. W. - Fetal placental vascular responses to prostacyclin after angiotensin II-induced vasoconstriction. Am. J. Physiol., 257: 102-107, 1989.
15 PARISI, V. M. \& WALSH, S. W. - Fetal vascular response to prostacyclin. Am. J. Obstet. Gynecol., 160 871-875, 1989.

16 PARK, M. K.; HAYASHI, S.; ROBOTHAN, J. L. - Coolinginduced contraction of guinea pig tracheal smooth muscle. Proc. Soc. Exp. Biol., 177: 283-289, 1984.

17 POWER, G. G. \& LONGO, L. D. - Sluice flow in the placenta: maternal vascular pressure effect on fetal circulation. Am. J. Physiol., 225: 1490-1496, 1973.

18 RITTENHOUSE, E. A.; HOHRI, H.; DILLARD, D. H.; MERENDINO, K. A. - Deep hypothermia in cardiovascular surgery. Ann. Thorac. Surg., 17: 63-98, 1974.

19 SCHWARTZ, D. B.; PHERNETTON, T. M.; STOCK, M. K.; RANKIN, J, H. G. - Placental vascular responses to 6-keto-prostaglandin E1 in the near-term sheep. Am. J. Obstet. Gynecol., 145: 406-410, 1983.

20 SOUHRADA, J. F.; PRESLEY, D.; SOUHRADA, M. - Mechanisms of the temperature effect on airway smooth muscle. Resp. Physiol., 53: 225-237, 1983.

21 THORNBURG, K. L.; BISSONETTE, J. M.; FABER, J. J. - Absence of fetal placenta waterfall phenomenon in chronically prepared fetal lambs. Am. J. Physiol., 230: 886-892, 1976.

22 VETH, A. F. L. \& VAN BEMMEL, J. H. - The role of the placental vascular bed in the fetal response to cord occlusion. In: LONGO, L. D. \& RENEAU, D. D. Fetal carciovascular physiology. New York, Garland Publishing, Inc., 1978. p. 579-604.

\section{Discussão}

\section{DR. OTONI MOREIRA GOMES \\ Belo Horizonte, MG}

Dr. Renato S. Assad, o senhor referiu variações aleatórias de parâmetros vetores da pesquisa como fluxo e pressão de perfusão, momento de administração e duração do efeito do nitroprussiato de sódio, que é geralmente fugaz. Numa investigação com apenas sete experimentos, essa aleatoriedade de vetores impede o alinhamento de resultados necessários à constituição do corpo científico do estudo. Isto prejudicou nosso engajamento solidário, durante a análise deste trabalho, que é original, atual, muito útil e promissor. As conclusōes e os efeitos obtidos pareceram-nos dependerem muito mais da vivência dos pesquisadores, nos diferentes momentos da investigação, do que das possibilidades oferecidas pela análise e estatística dos resultados expressos e redigidos. Três conclusōes principais foram destacadas pelo senhor: 1) a resistência vascular placentária (RVP) aumenta quando o fluxo de perfusão desce aquém de 150 $\mathrm{ml} / \mathrm{kg} / \mathrm{min}$, ou com a redução da pressão de perfusão abaixo de $40 \mathrm{mmHg}$. Essa interpretação é semelhante à descrita por Replogle e Lillehei, em 1962, para a variação da resistência vascular sistêmica (RVS) durante a 
ASSAD, R. S.; LEE, F. Y.; BERGER, K.; HANLEY, F. I. - Cirurgia cardiaca fetal: características hemodinâmicas da placenta durante circulação extracorpórea. Rev. Bras. Cir. Cardiovasc., 6(1): 38-44, 1991.

CEC, razão pela qual, desde aquela data, preconiza-se a rotina de fluxo arterial entre 2.200 e $2.400 \mathrm{ml} / \mathrm{m}^{2} / \mathrm{sup}$. corp. em pequenos pacientes; 2) a RVP aumenta com a hipotermia. Essa conclusão é, também, absolutamente semelhante à estabelecida por Lopez-Bello e colaboradores e por OZ e colaboradores, em 1960, estudando a RVS com CEC hipotérmica. Aqui, uma das razōes para a manutençăo do fluxo de perfusảo durante hipotermia moderada, evitando-se somação de dois efeitos vasoconstritores: hipotermia e hipofluxo. Ademais, sabese que, abaixo de $26^{\circ} \mathrm{C}$, cessa o efeito vasoconstrictor da hipotermia. Assim, existe analogia total entre a resposta vascular placentária entendida nesta pesquisa e os conhecimentos sedimentados sobre comportamento do RVS durante perfusão artificial, normotérmica e hipotérmica. Esse fato é reforçado pela experiência com cirurgia cardiaca em gestantes, onde sabemos que a CEC estável, nāo complicada, é provavelmente um dos fatores menos nocivos ao feto, de todo o procedimento. Em nosso Serviço, foram operadas duas pacientes gestantes (2: e 3 : mês de gestação, respectivamente), com lesōes mitrais, que deram à luz conceptos normais a termo. Com relação à 3 : conclusão, de que as variações da RVP dependam do mecanismo da resistência de Starling, entendemos que problemas relevantes de metodologia da pesquisa prejudicam sua cristalização científica. Igualmente, de modo quase empírico, preferimos admitir que as variaçōes descritas na RVP, em tudo idênticas às da RVS durante CEC, tenham dependido de modificaçōes angiocinéticas na primeira zona de arterialização placentária e não de fenômenos extrínsecos na zona de microcirculação.

\section{DR. ASSAD}

\section{(Encerrando)}

Em primeiro lugar, gostaria de agradecer ao Dr. Otoni o seu comentário. A circulação do corpo humano apresenta um sistema complexo de controle do tônus vascular e do fluxo sangüíneo para diferentes órgão e tecidos. Os três maiores componentes deste sistema incluem mecanismos locais, neuronais e humorais. Por outro lado, a vasculatura feto-placentária é desprovida de inervação, além de não apresentar mecanismo autoregulador, o que a diferencia fisiológica e anatomicamente da circulação sistêmica. Por esses motivos, acreditamos que as alteraçōes da resistência placentária não dependam apenas de "modificações angiocinéticas na primeira zona de arterialização placentária". Tendo em vista o fato de que a principal causa de morbidade e mortalidade fetal após a CEC está relacionada à disfunção placentária por alteraçōes hemodinâmicas da mesma, e que pouco se entende a respeito das respostas hemodinâmicas da placenta à CEC, seria apropriado, no atual nivel de conhecimento, examinar a placenta isoladamente, com o objetivo de observar a resposta intrínseca da placenta em condiçōes de CEC. Em relação ao nitroprussiato de sódio, sua infusão foi mantida constante durante todo o experimento, após o ajuste inicial da dose ideal, independentemente de variação do fluxo placentário. Portanto, sua ação "fugaz" foi controlada pela sua infusão contínua através de uma bomba. A relação inversamente proporcional entre fluxo e resistência em condiçōes de baixo fluxo e/ou baixa pressão de perfusão placentária foi uniformemente observada em todos os experimentos. Como as curvas e cada experimento foram estatisticamente significantes $(p<0,003)$, consideramos o número de experimentos realizados (sete) o suficiente para se obterem resultados estatisticamente significantes. Portanto, as conclusões aqui expostas foram baseadas nos dados concretos de análise de regressão linear expressas nos gráficos apresentados. Sobre a hipotermia como não haviam sido demonstradas anteriormente essas alteraçōes nesse peculiar leito vascular placentário, é prudente a pesquisa dessa condição durante a CEC. $\mathrm{O}$ aumento da resistência vascular placentária durante a hipotermia pode ser explicado principalmente pelo aumento da viscosidade sangüínea. Para temperatura abaixo de $26^{\circ} \mathrm{C}$, a viscosidade sangüínea seria maior ainda, o que proporcionaria maior resistência vascular placentária, uma vez que o tônus vascular placentário foi minimizado pela administração de um vasodilatador direto da musculatura lisa vascular. A circulação placentária é dividida em duas partes distintas: a materno-placentária e a feto-placentária. $O$ estudo realizado avalia o comportamento hemodinâmico da circulação feto-placentária. Em relação à circulação materno-placentária, é sabido que a vitalidade fetal durante a cirurgia cardiaca em gestantes está diretamente relacionada a uma CEC estável, isto é, aporte normal de oxigênio, nutrientes e remoção de metabólitos tóxicos da circulação fetal, garantidos por uma boa pressão de perfusão e saturação de $\mathrm{O}_{2}$ adequada durante a CEC. Estudos de Kaufmann, publicados em 1985, no periódico Contributions to Gynecology and Obstetrics, mostram que a isquemia de um a dois minutos da circulação maternoplacentária é seguida de alteraçōes ultra-estruturais severas da placenta, confirmando a absoluta necessidade de uma pressāo de perfusāo e de saturaçāo de $\mathrm{O}_{2}$ adequadas durante a intervenção materna. Quanto ao mecanismo da resistência de Starling, o qual foi exaustivamente discutido, achamos que outros mecanismos de aumento da resistência vascular durante o baixo fluxo e/ou pressão de perfusão podem também estar presentes. Qualquer que seja o mecanismo, a principal mensagem deste trabalho reside no fato de que a normotermia e um fluxo e/ou pressāo de perfusão umbilical adequados são primordiais para manter a resistência placentária baixa, vital para a manutenção das trocas gasosas e, conseqüentemente, para a sobrevida fetal durante e após a cirurgia cardiada. Muito obrigado. 